J. Lake Sci. (湖泊科学), 2013, 25(2): 243-249

http: //www. jlakes.org. E-mail : jlakes@niglas.ac.cn

(C) 2013 by Journal of Lake Sciences

\title{
湖泊沉积物粒度分析前处理过程中絮凝现象的消除方法研究”
}

\author{
胡宗杰 ${ }^{1,2}$, 颜佳新 ${ }^{1,2 * *}$, 韩鸿印 ${ }^{2}$ \\ (1: 中国地质大学 (武汉) 地球科学学院, 武汉 430074) \\ (2: 中国地质大学 (武汉) 生物地质与环境地质国家重点实验室, 武汉 430074)
}

\begin{abstract}
摘 要: 采用激光粒度仪对湖泊沉积物进行粒度分析时需要进行必要的前处理. 在有效去除有机质与碳酸盐颗粒的同 时, 需要消除细粒泥沙质样品中絮凝现象对粒度分析结果造成的误差或错误. 本文以武汉三角湖表层沉积物样品为例, 采用 X 射线粉晶衍射 (XRD) 对其矿物组成和粘土矿物种属进行鉴定, 并采用全自动激光粒度仪对样品进行粒度分析. $\mathrm{X}$ 射线粉晶衍射结果表明样品中非粘土矿物主要为石英与长石, 粘土矿物以伊利石、高岭石、绿泥石为主; 粒度分析结果显 示样品中小于 $30 \mu \mathrm{m}$ 组分占 $94.5 \%$. 这些特征表明该样品易于在前处理过程中形成絮凝. 本次研究采用单因素寻优法探 讨了超声震荡、分散剂、洗酸对于以伊利石、高岭石、绿泥石为主、平均粒径较小的样品消除絮凝现象的最佳条件. 研究结 果表明, 针对此类样品消除絮凝现象的最佳条件为: 超声震荡时间应选择 $6 \sim 8 \mathrm{~min}$, 分散剂浓度为 $20 \mathrm{~g} / \mathrm{L}$ 左右, 同时在粒 度分析之前需要对样品进行洗酸.
\end{abstract}

关键词: 湖泊沉积物; $\mathrm{X}$ 射线粉晶衍射; 激光粒度分析; 前处理; 絮凝; 消除方法; 三角湖

\section{Eliminating flocculation in the pretreatment of lacustrine deposits for particle size analysis}

\author{
HU Zongjie ${ }^{1,2}$, YAN Jiaxin ${ }^{1,2}$ \& HAN Hongyin ${ }^{2}$ \\ (1: Faculty of Earth Sciences, China University of Geosciences, Wuhan 430074, P. R. China) \\ (2: State Key Laboratory of Biogeology and Environmental Geology, China University of Geosciences, Wuhan 430074, P. R. \\ China)
}

\begin{abstract}
Lake sediment should be pretreated before particle size analysis. It is necessary to eliminate the error caused by flocculation phenomenon of fine grain of sediment samples at the same time of effectively removing organic matter and carbonate particles. Surface sediment sample from the Lake Sanjiao in Wuhan was used for X-ray powder diffraction (XRD) to identify its mineral composition and clay species and automatic laser granulometer for grain size analysis. The XRD result demonstrated that the main nonclay minerals in sample were quartz and feldspar; while the clay minerals were primarily illite, kaolinite, chlorite. Furthermore, the result of grain size analysis showed that the grain size of $94.5 \%$ of component in sample was less than $30 \mu \mathrm{m}$. These characteristics made the sample easy to form flocculation at the pretreatment process of grain size analysis. Based on the results above, for the sample which are composited primarily with illite, kaolinite, chlorite, a single factor optimization method including ultrasonic concussion, dispersing agent and acid washing was used to check the optimum condition for eliminating flocculation. Results indicate that the optical condition for eliminating flocculation are ultrasonic concussion time: $6-8$ min, dispersing agent concentration: $20 \mathrm{~g} / \mathrm{L}$, with acid washing before particle size analysis.
\end{abstract}

Keywords: Lake sediment; X-ray powder diffraction; laser granulometer; pretreatment; flocculation; eliminate method; Lake Sanjiao

沉积物的粒度数据被广泛应用于区分沉积环境、判定物质运输方式、判别水动力条件、分析粒径趋势等 方面 $^{[1-4]}$, 湖泊沉积物是记录湖泊及其流域气候环境信息的有效载体, 具有连续性好、分辨率高、包含信息量

\footnotetext{
* 国家自然科学基金项目 (41072078) 和武汉新区六湖水系网络工程三角湖与北太子湖底质水质特征分析项目 (2009316236) 联合资助. 2012-05-14 收稿;2012-08-31 收修改稿. 胡宗杰, 男, 1984 年生, 硕士; E-mail: huzongjie84@163.com.

** 通信作者;E-mail:jaxy2008@163.com.
} 
丰富等特点, 因而成为恢复古环境演化特征的重要指标, 更是古气候重建的重要载体 ${ }^{[5-7]}$. 目前广泛采用的 激光粒度分析方法, 相对于传统的方法如沉降法、篮析法、移液管法、电阻法和影像分析法等, 不但操作简 便、时效性高、智能化, 测量范围更为宽泛, 而且精度大幅提高 ${ }^{[2,8-9]}$. 采用激光粒度仪进行粒度测试首先要对 样品进行前处理, 目的是在不造成颗粒破坏与损失的前提下, 去除有机质和碳酸盐, 同时消除絮凝现象对结 果的影响. 而不同的前处理方法会对分析结果造成明显影响 ${ }^{[2,10-17]}$.

样品粘土矿物组成与粒度分布状况是絮凝产生的主导因素 ${ }^{[18]}$. 目前有学者分别探讨了粘土矿物组成、 粒度分布状况与絮凝之间的关系. 冯志刚等 ${ }^{[12]}$ 对富含蒙脱石、三水铝石的红色风化壳粒度分析前处理方法 做了研究, 认为高浓度盐酸处理会造成蒙脱石晶格破坏, 导致矿物分解和硅胶体出溶, 会对矿物产生胶结现 象, 从而造成颗粒凝聚, 矿物粒度变粗, 伊利石、高岭石、绿泥石等矿物对盐酸无明显敏感性 ${ }^{[12]}$. 也有学者对 细粒泥沙的絮凝现象和机理进行了深人的研究 ${ }^{[19-20]}$, 探讨了容易造成絮凝的最大颗粒粒径, 目前广泛接受 $30 \mu \mathrm{m}$ 是静水条件下形成絮凝的极限粒径 ${ }^{[21]}$, 还有学者得出伊利石等粘土矿物的存在有利于絮凝作用以桥 联形式形成络合物 ${ }^{[11]}$.

对样品进行超声震荡、加人分散剂、洗酸是消除絮凝的有效方法, 不同样品应针对性地选择不同方法. 粒度分析前处理过程中试剂对于以蒙脱石、三水铝石等粘土矿物成分为主样品的影响已经有较多研究 ${ }^{[12]}$, 而对于主要成分为伊利石、高岭石、绿泥石等的粘土矿物, 粒径分布较小的湖泊沉积物或其他类似组分沉积 物 (如黄海沉积物), 其前处理过程中的絮凝现象消除方法还未见报道. 本文结合 X 射线粉晶衍射 (XRD) 分 析结果及粒度分布状况, 首先确定样品的矿物学特征, 然后从湖泊沉积物粒度分析前处理方法对絮凝现象 的消除着手, 采用单变量寻优法探讨了各种因素 (主要为超声震荡时间、分散剂浓度、洗酸与否) 对结果的 影响.

\section{1 样品及实验方法}

\section{1 样品的来源}

样品选自武汉市六湖连通工程中主要湖泊之一的三角湖 $\left(30^{\circ} 14^{\prime} \mathrm{N}, 114^{\circ} 10^{\prime} \mathrm{E}\right.$; 图 1) 底质表层样. 三角湖 湖长 $2.6 \mathrm{~km}$, 最大湖宽 $1.8 \mathrm{~km}$, 平均湖宽 $0.7 \mathrm{~km}$, 平均水深 $2 \mathrm{~m}$, 湖泊面积 $1.8 \mathrm{~km}^{2}$, 属于外流淡水湖 ${ }^{[22]}$, 湖区 属于北亚热带季风气候, 湖水补给主要为地表径流和大气降水.

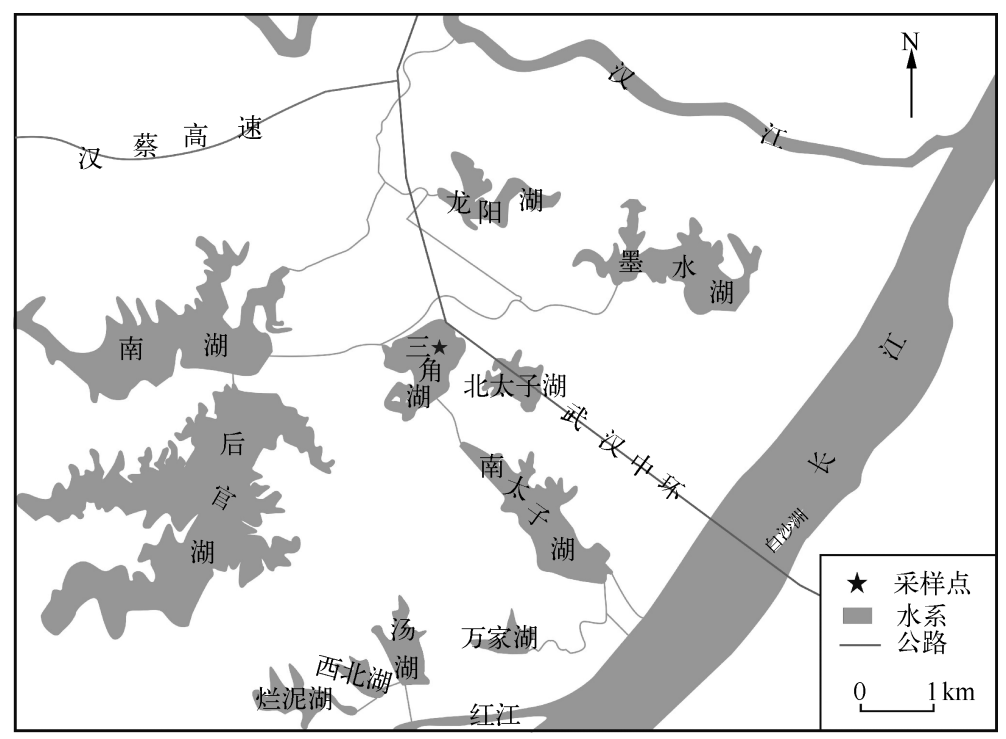

图 1 三角湖底质沉积物采样点位置

Fig. 1 Location of surficial sediment sampling in the Lake Sanjiao 


\section{2 仪器与试剂}

$X$ 射线粉晶衍射 (XRD) 分析测试在中国地质大学 (武汉) 地质与矿产资源国家重点实验室的荷兰帕纳 科 X'Pert Pro 型 $\mathrm{X}$ 射线粉晶衍射仪上进行, 人射光源为 $\mathrm{CuK} \alpha$ 辐射, $\mathrm{Ni}$ 片滤波, $\mathrm{X}$ 光管工作电压为 $40 \mathrm{kV}$, 电 流为 $40 \mathrm{~mA}$; 光阑系统为 $\mathrm{DS}=\mathrm{SS}=1^{\circ} ; \mathrm{RS}=0.3 \mathrm{~mm}$. 使用连续扫描方式, 扫描速度为 $8^{\circ} / \mathrm{min}, 2 \theta$ 分辨率为 $0.02^{\circ}$. 扫描范围为 $3^{\circ} \sim 65^{\circ}$, 采用超能探测器.

粒度分析测试在中国地质大学 (武汉) 生物地质与环境地质国家重点实验室完成, 采用美国 Beckman Coulter 公司的 LS230 型全自动激光粒度分析仪. 仪器测试范围为 $0.04 \sim 2000 \mu \mathrm{m}$. 重复测量误差 $< \pm 1 \%$. 超 声震荡测试采用中国昆山超声仪器有限公司生产的 KQ-250DB 型数控超声波清洗器. 超声频率 $40 \mathrm{kHz}$, 超声 功率 $250 \mathrm{~W}$, 功率可调 40\% 100\% , 时间可调 $1 \sim 480 \mathrm{~min}$.

主要试剂:乙二醇、双氧水、盐酸、六偏磷酸钠、去离子水.

\section{3 样品处理和分析方法}

样品的矿物组成和粘土矿物种属鉴定分别采用粉晶片和粘粒定向片. 粉晶片采用新鲜原样, 首先将原 样置于干燥箱中在 $60^{\circ} \mathrm{C}$ 下烘干, 然后置于玛瑙研钵中研磨, 过 300 目笁, 最后将过篎微米粒级颗粒置于带有 凹槽的玻璃板中压实刮平即可. 粘粒定向片制备具体流程如下: 取 $15 \sim 20 \mathrm{~g}$ 上述微米粒级原样颗粒于大烧 杯中, 加适量的蒸馏水摚拌 $45 \mathrm{~min}$, 采用斯托克斯沉降法将样品静置 $8 \mathrm{~h}$, 然后采用虹吸法获得烧杯上层 $10 \mathrm{~cm}$ 水溶液, 于离心机中分离得到粒径 $<2 \mu \mathrm{m}$ 的粘土样品. 将其涂布于玻璃片上, 自然干燥固结后得到自 然定向片 ( NG), 将自然定向片置于干燥血. 中, 在 $70^{\circ} \mathrm{C}$ 的乙二醇饱和蒸汽中饱和 $3 \mathrm{~h}$ 可制成乙二醇饱和片 (EG). 分别将粉晶片、自然定向片、乙二醇饱和片上机测试.

本文采用单变量寻优法, 分别测定超声振荡时间、洗酸与否、分散剂六偏磷酸钠 $\left(\mathrm{Na}_{3} \mathrm{PO}_{4}\right)_{6}$ 浓度对消除 絮凝的作用. 前处理方法描述如下:

方法 A: 目的是比较不同超声振动时间对消除絮凝的作用. 取 $1 \mathrm{~g}$ 样品 6 份, 分别放人 $50 \mathrm{ml}$ 烧杯中, 加 人 $10 \% \mathrm{H}_{2} \mathrm{O}_{2} 10 \mathrm{ml}$, 去除有机质, 反应 $24 \mathrm{~h}$, 吸取上清液, 加人去离子水清洗, 吸取上清液; 然后加人 $10 \% \mathrm{HCl}$ $10 \mathrm{ml}$, 去除碳酸盐, 反应 $24 \mathrm{~h}$, 吸取上清液, 再加人去离子水反复洗酸, 直至溶液接近中性, 吸取上清液, 之后 加人分散剂 $20 \mathrm{~g} / \mathrm{L}\left(\mathrm{Na}_{3} \mathrm{PO}_{4}\right)_{6}$, 分别给予 $2 、 4 、 6 、 8 、 10 、 12 \mathrm{~min}$ 超声震荡后上机测试.

方法 B: 目的是对比洗酸与否对消除絮凝的作用. a: 取 $1 \mathrm{~g}$ 样品, 放人 $50 \mathrm{ml}$ 烧杯中, 加人 $10 \% \mathrm{H}_{2} \mathrm{O}_{2}$ $10 \mathrm{ml}$, 去除有机质, 反应 $24 \mathrm{~h}$, 吸取上清液, 加人去离子水清洗, 吸取上清液; 然后加人 $10 \% \mathrm{HCl} 10 \mathrm{ml}$, 去除 碳酸盐, 反应 $24 \mathrm{~h}$, 吸取上清液, 之后加人分散剂 $20 \mathrm{~g} / \mathrm{L}\left(\mathrm{Na}_{3} \mathrm{PO}_{4}\right)_{6}$ 超声震荡 $7 \mathrm{~min}$ 使颗粒充分分散, 上机测 试. b: 在 $\mathrm{a}$ 的基础上将用盐酸处理后的样品, 反应完毕静置 $24 \mathrm{~h}$ 后, 首先将盐酸等废液用一次性吸管吸取至 最低值, 此后向其中加人 $30 \mathrm{ml}$ 去离子水, 所用去离子水 $\mathrm{pH}$ 为 6.22 ( $\mathrm{pH}$ 在 $6 \sim 8$ 之间均可认为溶液接近中 性), 静置 $8 \mathrm{~h}$ 以上, 将上清液吸出, 反复以上过程, 直至溶液基本接近中性.

方法 $\mathrm{C}$ : 目的是探讨分散剂 $\left(\mathrm{Na}_{3} \mathrm{PO}_{4}\right)_{6}$ 浓度对消除絮凝的作用. 取 $1 \mathrm{~g}$ 样品 6 份, 放人 $50 \mathrm{ml}$ 烧杯中, 加人 $10 \% \mathrm{H}_{2} \mathrm{O}_{2} 10 \mathrm{ml}$, 去除有机质, 反应 $24 \mathrm{~h}$, 吸取上清液, 加人去离子水清洗, 吸取上清液; 然后加人 $10 \% \mathrm{HCl}$ $10 \mathrm{ml}$, 去除碳酸盐, 反应 $24 \mathrm{~h}$, 吸取上清液, 再加人去离子水反复洗酸, 直至溶液接近中性, 吸取上清液, 之后 分别加人分散剂 $0 、 5 、 10 、 15 、 20 、 25 、 30 、 35 、 40 \mathrm{~g} / \mathrm{L}\left(\mathrm{Na}_{3} \mathrm{PO}_{4}\right)_{6}$, 超声震荡 $7 \mathrm{~min}$ 使颗粒充分分散后上机测试.

本文还对以上各方法进行了重复实验, 样品的平行测定误差均在 $2 \%$ 以内.

\section{2 结果与讨论}

\section{1 三角湖样品矿物学及粒度特征}

粉晶片的 $X$ 射线粉晶衍射 (图 2a) 分析结果表明, 三角湖样品中主要由石英 ( $4.24 \AA 、 3.33 \AA$ )、长石 $(3.24 \AA 、 3.19 \AA)$ 、方解石 $(3.02 \AA)$ 、伊利石 $(10.0 \AA 、 5.0 \AA 、 3.33 \AA)$ 、高岭石 $(7.1 \AA 、 3.58 \AA)$ 及绿泥石 $(14.2 \AA$ 、 $7.1 \AA 、 4.84 \AA 、 3.53 \AA$ ) 等组成. 对分离样品进行物相分析后, 通过样标法 ${ }^{[23]}$ 确定了矿物的相对含量, 其中非粘 土矿物 (石英与长石) 占 $35 \%$, 粘土矿物含量约占 $65 \%$.

提纯的粘土矿物试样自然定向片及相应乙二醇饱和片的 X 射线粉晶衍射结果 (图 $2 \mathrm{~b}$ ) 表明, 三角湖样 品中粘土矿物主要有伊利石 $(10.0 \AA 、 5.0 \AA 、 3.33 \AA$ )、高岭石 $(7.15 \AA 、 3.58 \AA)$ 及绿泥石 $(14.2 \AA 、 7.1 \AA 、 4.72 \AA$ 、 
$3.53 \AA$ ). 在自然定向片中 $15 \sim 17.5 \AA$ 未出现明显峰值, 经乙二醇饱和处理后, 同样未观察到此位置出现峰或 峰值变宽，因此可判定样品中不存在蒙脱石或伊利石/蒙脱石混层矿物. 通过衍射峰的面积计算法 ( $100 \%$ 法) ${ }^{[24-25]}$ 得出样品中粘土矿物伊利石含量最高, 占 $74 \%$ (约占样品总量的 $49 \%$ ), 高岭石 $13 \%$ (约占样品总 量的 $8 \%$ ), 绿泥石 $13 \%$ (约占样品总量的 $8 \%$ ), 不含蒙脱石或伊利石/蒙脱石混层矿物.
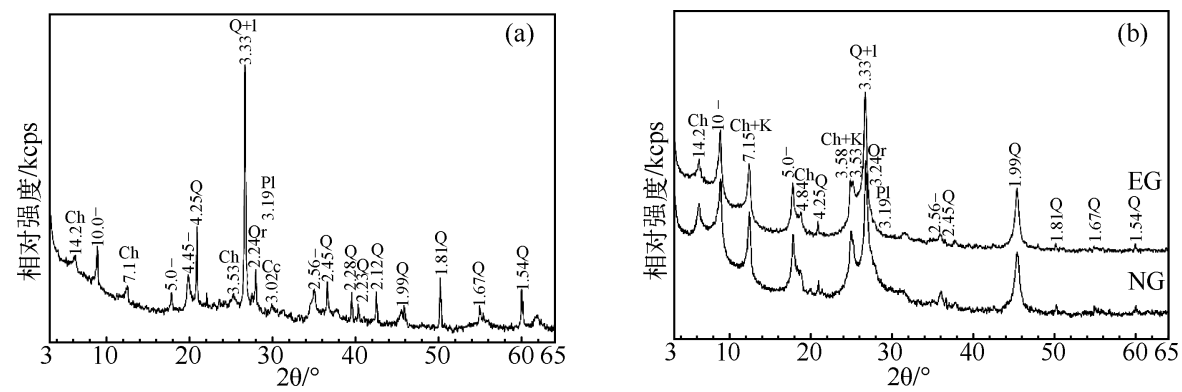

图 2 三角湖样品粉晶衍射图: 样品粉晶片衍射图 (a); 样品粘粒定向片衍射图 (b) ( Q-石英; Or一正长石; $\mathrm{Pl}$ 一斜长石; I一伊利石; $\mathrm{Ch}$ 一绿泥石; $\mathrm{K}$ 一高岭石; Cc一方解石; $\mathrm{NG}$ 一自然粘粒定向片; EG一乙二醇饱和粘粒定向片）

Fig. 2 XRD patterns of Lake Sanjiao samples: XRD patterns of powder samples(a);XRD patterns of oriented samples (b) ( Q-Quartz; Or-Orthoclase; Pl-Plagioclase; I-Illite; Ch-Chlorite;K-Kaolinite; Cc-Calcite; NG-Oriented air-dried samples; EG-Oriented ethylene glycol saturated clay samples)

从方法 A 中超声振荡 $6 \mathrm{~min}$ 得出的样品粒度分布特征可以看出, 样品粒度呈现双峰分布 (图 3). 双峰模 式在湖泊沉积物粒度研究中较为常见, 一般认为是两种不同动力机制对沉积作用的影响造成的, 如孙千里 等 ${ }^{[26]}$ 揭示岱海沉积物粒度分布呈现双峰模式为风力与流水作用的影响.

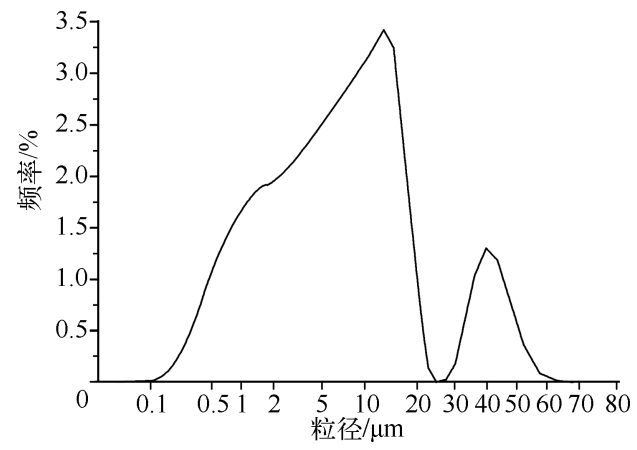

图 3 三角湖粒度频率分布特征

Fig. 3 Distribution of grain size frequencies of Lake Sanjiao samples

样品粒径大部分小于 $30 \mu \mathrm{m}$ (占 $94.5 \%$ ), 粒径大于 $30 \mu \mathrm{m}$ 组分仅占 $5.5 \%$, 大部分颗粒粒径分布在可产生絮 凝现象的范围内 (小于30 $\mu \mathrm{m}$ ), 易产生絮凝现象. 为了更 好的确定大于 $30 \mu \mathrm{m}$ 组分的确切含量, 同时区分絮凝现 象对实验结果的干扰, 本文还选取了篮析法与镜下观察 法对结果进行分析与论证. 篮析法 3 次重复实验得出样 品中大于 $30 \mu \mathrm{m}$ 组分的含量分别为 $5.2 \% 、 5.1 \%$ 和 $5.2 \%$, 这与激光粒度仪法得出的 5.5\% 存在很小偏差, 但基本一 致. 分析存在偏差的原因在于有长条状样品可以通过篮 孔, 而在采用激光粒度仪分析方法时, 由于采用了等体积 法不会造成此偏差. 镜下观察到大于 $30 \mu \mathrm{m}$ 组分主要为 石英, 同时有少量长条状云母. 石英颗粒最大直径可达 65 $\mu \mathrm{m}$ 左右, 未发现大于 $70 \mu \mathrm{m}$ 组分. 由此判断若实验结果 与以上数据存在较大偏差, 可认为是絮凝现象的干扰, 应

加以消除.

\section{2 粒度分析前处理过程各条件对消除絮凝的作用}

颗粒之间的物理化学作用可使得颗粒之间产生微观结构, 而泥沙颗粒愈细, 其比表面积愈大, 随着这种 细颗粒含量的增加, 相邻的若干带有吸附水膜的细颗粒便彼此连接在一起形成絮团, 这种现象称为絮凝现

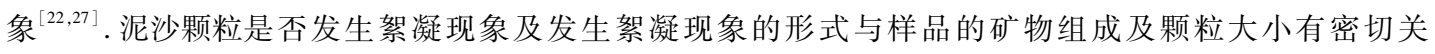
系 ${ }^{[19,27]}$. 从以上针对样品粘土矿物成分及粒度分析得出: 粘土矿物成分以伊利石、高岭石、绿泥石为主, 颗粒 粒度较小的样品容易以吸附、桥联、网捕等形式聚合成絮凝体, 在粒度分析测定结果的表现形式为出现多峰 现象或数据峰值产生变化. 
湖泊沉积物有机质可以作为配位体吸附载体, 和基本的金属离子和有机化合物结合, 从而导致絮凝现 象产生. 双氧水能够有效去除有机质, 同时具有抗絮凝作用, 进行前处理时被广泛采用 ${ }^{[17]}$. 由于采用样品量 较少 $(1 \mathrm{~g})$, 加人 $10 \mathrm{ml} 10 \% \mathrm{H}_{2} \mathrm{O}_{2}$ 可完全去除有机质, 剩余过量双氧水不会对结果造成明显影响, 对于粒径较 大的有机质, 可手动去除 ${ }^{[17]}$. 因此实验中可排除有机质颗粒对结果造成的影响.

盐酸通常被用来去除样品中的碳酸盐颗粒, 在湖泊沉积物粒度分析前处理时加人的盐酸量不足, 使碳 酸盐颗粒不能充分溶解, 但如果加人过量的盐酸, 可能使部分矿物与之快速发生反应, 如蒙脱石含量较高的 样品, 高浓度盐酸会造成硅胶体出溶, 对于以伊利石为主的样品, 则不会产生上述现象. 盐酸的用量还会影 响湖泊沉积物的 $\mathrm{pH}$, 较低的 $\mathrm{pH}$ 可导致或加重粘土絮凝颗粒产生, 对根据极限法计算, 加人 $10 \mathrm{ml} 10 \% \mathrm{HCl}$ 反应完全后依然会有剩余盐酸存在,同时溶液呈酸性,部分金属阳离子的存在也会加重絮凝现象.

因此有必要在针对湖泊沉积物粒度分析前处理之前对絮凝加以消除.

2.2.1 超声震荡时间对消除絮凝的作用 在进行湖泊沉积物粒度分析的前处理时, 相对于手动摇晃分散样 品, 对固结性较强的样品进行超声震荡是必要的. 超声时间过短, 凝聚的颗粒不能有效分散, 而时间过长会导 致颗粒被击碎; 随着时间的加长, 增加更多细粒粘土颗粒, 又会造成絮凝现象, 这都将影响数据的可靠性. 有研 究者通过实验发现在所有单一的前处理方法中,超声波的分散效果最好,但是时间必须进行控制 ${ }^{[10,28-29]}$.

采用不同超声震荡时间对样品进行前处理对粒度测试结果产生很大影响, 不同超声条件下样品平均粒 径数值差异较大, $2 \mathrm{~min}$ 时平均粒径为 $10.14 \mu \mathrm{m}, 12 \mathrm{~min}$ 时平均粒径为 $7.42 \mu \mathrm{m}$ (图 4). 超声时间过短 (2 min) 或者过长 (12 min) 都会有絮凝现象存在 (图 4a), 超声时间过短, 沉积物颗粒不能有效分散, 随着超 声时间的加长, 沉积物平均粒径变小. 在超声低于 $6 \mathrm{~min}$ 时, 粒径减小速率明显大于超过 $8 \mathrm{~min}$ 的速率, 这是 由于超声低于 $6 \mathrm{~min}$ 时絮凝颗粒以分散为主, 超声 $8 \mathrm{~min}$ 达到粒径破碎临界值, 此时颗粒以破碎为主, 同时需 要更大强度能量才能将颗粒再次击碎. 鹿化煜等 ${ }^{[15]}$ 对风成红粘土粒度测量中较多细颗粒的增多做出了同样 的解释. 因此超声 $8 \mathrm{~min}$ 为颗粒破碎的临界值. 在超声 $6 \mathrm{~min}$ 到 $8 \mathrm{~min}$ 时平均粒径变化趋于水平, 可以认为此 时颗粒处于真实值状态.

(a)

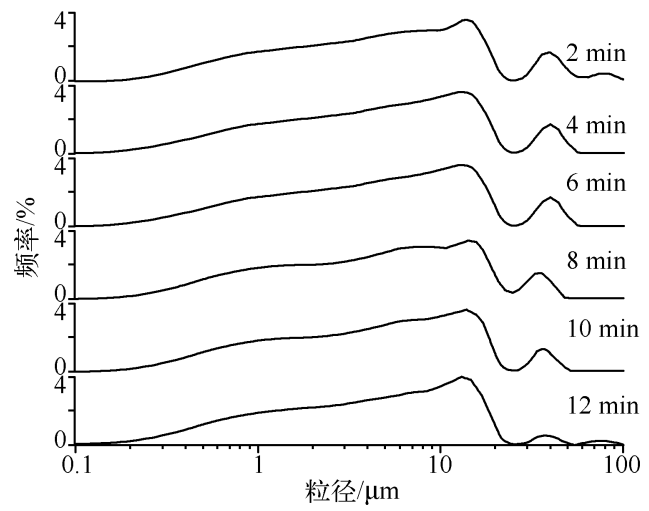

(b)

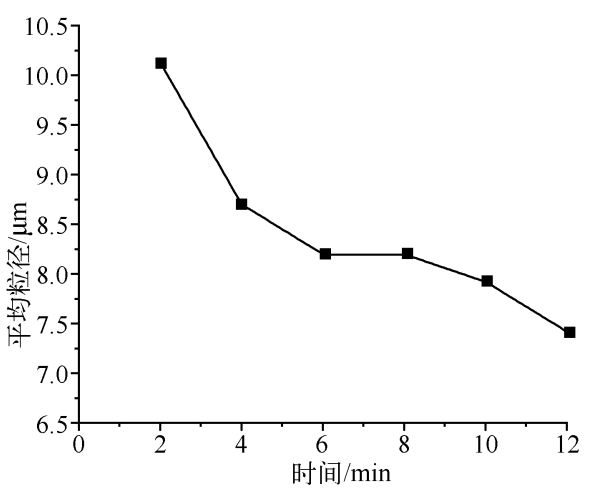

图 4 超声震荡对实验结果的影响: 不同超声震荡时间对粒度影响 (a); 粒度随超声震荡时间变化趋势 (b)

Fig. 4 The effect of ultrasonic concussion on the experimental results: Impact of different ultrasound concussion time for grain size (a); Change of grain size with the ultrasonic concussion time(b)

2.2.2 多余盐酸洗酸与否对消除絮凝的作用 沉积物 $\mathrm{pH}$ 是影响絮凝的主要因素, 用盐酸除去碳酸盐后, 如 果不将剩余盐酸清洗干净, 残存的 $\mathrm{HCl}$ 不但会腐蚀试验仪器, 而且有可能再次产生絮凝 ${ }^{[1,30-31]}$. 洗酸与否可 导致粒度分布状况发生明显变化, 不洗酸的结果比洗酸的结果多出一个峰, 粒度数值总体偏大(图 5 ). 该现 象的出现可解释为未经洗酸, 样品的 $\mathrm{pH}$ 过低, 导致颗粒产生絮凝; 经洗酸后, 样品的 $\mathrm{pH}$ 上升, 颗粒不易产生 絮凝. 所以在用盐酸处理后, 应加人去离子水进行洗酸.

本文在实验过程中采用 $\mathrm{pH}$ 计对每次操作过程中变化进行了测定, 4 次洗酸后溶液 $\mathrm{pH}$ 分别为 0.42 、 


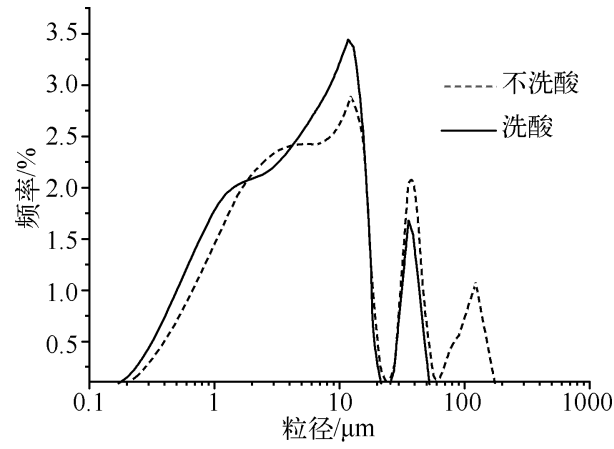

图 5 洗酸对实验结果的影响

Fig. 5 The effect of acid washing on the experimental results

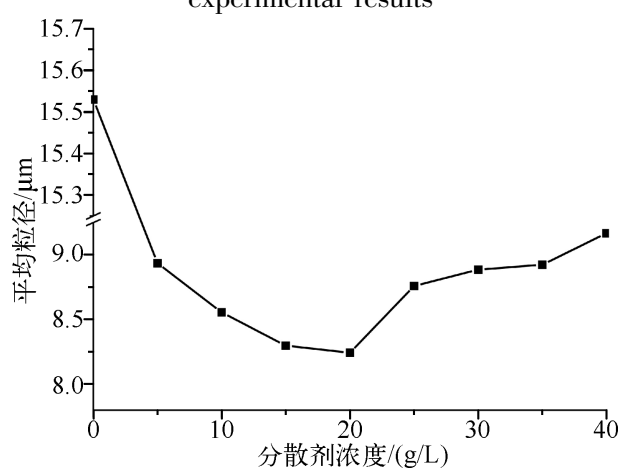

图 6 平均粒径随分散剂浓度变化曲线

Fig. 6 Change of mean grain size with the dispersant concentration curve
$1.50 、 2.70$ 和 6.04 , 最后已接近中性, 不会对实验结果产 生影响,也不会对仪器造成腐蚀.

2.2.3 分散剂对消除絮凝的作用 $\left(\mathrm{Na}_{3} \mathrm{PO}_{4}\right)_{6}$ 可以有效分 散沉积物颗粒, 防止絮凝现象产生 ${ }^{[4]}$, 目前被普遍采用, 针 对不同样品应该选用不同浓度的 $\left(\mathrm{Na}_{3} \mathrm{PO}_{4}\right)_{6}$ 进行分散. Ghilardi 等 $^{[4]}$ 在进行沉积柱样品粒度分析时采用 $5 \mathrm{~g} / \mathrm{L}$ 的 $\left(\mathrm{Na}_{3} \mathrm{PO}_{4}\right)_{6}$ 分散, 王君波等 ${ }^{[10]}$ 提出对湖泊沉积物进行粒度 分析时加人 $10 \mathrm{ml} 0.05 \mathrm{~mol} / \mathrm{L}($ 约 $30 \mathrm{~g} / \mathrm{L})\left(\mathrm{Na}_{3} \mathrm{PO}_{4}\right)_{6}$ 可降 低样品的凝聚性, 增强超声振荡对样品的撞击作用, 使得 实验结果由单峰呈现双峰, 使用时需有选择性.

从方法 C 测定结果( 图 6) 可以得出: (1) 验证了分散剂 浓度为 $20 \mathrm{~g} / \mathrm{L}$ 左右, 超声震荡时间在 $6 \sim 8 \mathrm{~min}$ 粒径变化 趋于水平, 选择 $7 \mathrm{~min}$ 的合理性; (2) 不加人分散剂与加人 量的多少都会对测试结果中平均粒径得变化产生影响明 显; 3 沉积物粒径随着分散剂浓度增加先降低(分散剂浓 度 $0 \sim 2 \%$ ) 后增加 ( 分散剂浓度 $2 \% \sim 4 \%$ ), 浓度较低时, 絮凝颗粒不能有效分散, 三角湖沉积物粒径在 $\left(\mathrm{Na}_{3} \mathrm{PO}_{4}\right)_{6}$ 浓度为 $20 \mathrm{~g} / \mathrm{L}$ 时出现拐点, 分散效果最佳. 随着浓度的进 一步增大, $\left(\mathrm{Na}_{3} \mathrm{PO}_{4}\right)_{6}$ 的粘性增强, 颗粒再次粘合, 导致平 均粒径再次增大 ${ }^{[32]}$.

\section{3 结论}

$X$ 射线粉晶衍射分析结果表明, 武汉三角湖底层沉积 物由石英、长石以及粘土矿物组成, 粘土矿物以伊利石、 高岭石、绿泥石为主; 全自动激光里粒度仪对样品进行粒 度分析结果显示, 样品中小于 $30 \mu \mathrm{m}$ 组分约占 $94.5 \%$, 这

些特征表明该类样品易于在粒度分析前处理过程中形成絮凝.

针对此类样品, 本文采用单变量寻优法, 综合探讨了在粒度分析前处理过程中, 在有效去除有机质与碳 酸盐颗粒的同时, 不同超声震荡时间、不同分散剂浓度、洗酸与否对絮凝现象消除的影响. 本研究结果表明, 对此类样品超声震荡应选择 $6 \sim 8 \mathrm{~min}$, 超声时间过短不能有效分散颗粒, 时间过长会将原生颗粒击碎, 细小 颗粒增多, 加重絮凝; 分散剂浓度应选用 $20 \mathrm{~g} / \mathrm{L}$ 左右, 浓度过低导致分散效果不佳, 加人过量, 六偏磷酸钠粘 性增强, 颗粒重新凝聚; 对样品采用盐酸前处理后需要洗酸, 既可以避免残余盐酸对仪器造成的腐蚀, 又可 有效消除絮凝对实验结果造成的影响.

本方法对于以伊利石、高岭石、绿泥石为主的粘土矿物, 以及粒径普遍小于 $30 \mu \mathrm{m}$ 易于发生絮凝现象的 细粒沉积物样品粒度分析前处理过程絮凝现象的消除提供了一定参考与借鉴.

\section{4 参考文献}

[ 1 ] 戴东林,陈庸勋. 沉积岩(物) 粒度分析及其应用. 北京: 地质出版社,1976.

[ 2 ] Allen JRL, Thornley DM. Laser granulometry of Holocene estuarine silts: effects of hydrogen peroxide treatment. The Holocene, 2004, 14(2) : 290-295.

[ 3 ] Chen Z, Stanley DJ, Wright EE. Selective sorting, storage and progressive dilution of sediment in two tropical deltas, Veracruz, Mexico. Journal of Coastal Research, 2000, 16(2): 470-481.

[ 4 ] Ghilardi M, Kunesch S, Styllas M et al. Reconstruction of Mid-Holocene sedimentary environments in the central part of the Thessaloniki Plain (Greece), based on microfaunal identification, magnetic susceptibility and grain-size analyses. Geomorphology, 2008, 97(3/4) : 617-630. 
[ 5 ] Campbell C. Late Holocene lake sedimentology and climate change in southern Alberta, Canada. Quaternary Research, $1998,49(1): 96-101$.

[ 6 ] Sun Q, Zhou J, Shen J et al. Environmental characteristics of Mid-Holocene recorded by lacustrine sediments from Lake Daihai, north environment sensitive zone, China. Science in China Series D: Earth Sciences, 2006, 49(9): 968-981.

[ 7 ] Prokopenko AA, Kendall CSGC. Seismic evidence for the Pleistocene depositional changes in Lake Hovsgol, Mongolia, and implications for the age model and the sediment grain size record of KDP-01 drill core. Journal of Paleolimnology, $2008, \mathbf{4 0}(4)$ : 1185-1192.

[ 8 ] Beuselinck L, Govers G, Poesen J et al. Grain-size analysis by laser diffractometry: comparison with the sieve-pipette method. Catena, 1998, 32(3/4) : 193-208.

[ 9 ] Konert M, Vandenberghe J. Comparison of laser grain size analysis with pipette and sieve analysis: a solution for the underestimation of the clay fraction. Sedimentology, 1997, 44(3): 523-535.

[10] 王君波,朱立平. 不同前处理对湖泊沉积物粒度测量结果的影响. 湖泊科学, 2005,17(1):17-23.

[11] 王德杰,范代读. 不同预处理对沉积物粒度分析结果的影响. 同济大学学报: 自然科学版, 2003,31(3):314-318.

[12] 冯志刚, 王世杰,罗维均等. 不同前处理方法对红色风化壳粒度测试结果的影响. 矿物学报,2006,26(1):1-7.

[13] 张红艳, 鹿化显, 赵 军等. 超声波振荡对细颗粒黄土样品粒度测量影响的实验分析. 沉积学报, 2008,26(3): 494-500.

[14］贾红娟, 秦小光, 刘嘉麒. 激光粒度仪分析结果中假峰形成的原因及消除方法. 海洋地质与第四纪地质, 2009, (5): $145-152$.

[15] 鹿化煜, 苗晓东. 前处理步骤与方法对风成红粘土粒度测量的影响. 海洋地质与第四纪地质, 2002, 22 (3): 129-135.

[16] 孙有斌,高 抒. 前处理方法对北黄海沉积物粒度的影响. 海洋与湖沼,2001,32(6):665-671.

[17] Gray AB, Pasternack GB, Watson EB. Hydrogen peroxide treatment effects on the particle size distribution of alluvial and marsh sediments. The Holocene, 2010, 20 (2) : 293-301.

[18 ] Sekiou F, Kellil A. Effect of organic and mineral matters on kinetic and performance of flocculation. Desalination, 2009, 249(2) : 891-894.

[19] Krishnappan BG, Marsalek J. Modelling of flocculation and transport of cohesive sediment from an on-stream stormwater detention pond. Water Research, 2002, 36(15) : 3849-3859.

[20] Nikora V, Aberle J, Green M. Sediment flocs: Settling velocity, flocculation factor, and optical backscatter. Journal of Hydraulic Engineering, 2004, 130(10) : 1043-1047.

[21] 王党伟, 杨国录, 余明辉. 静水中粘性细颗粒泥沙絮凝临界粒径的确定及其影响因素分析. 泥沙研究, 2009, (1): 74-80.

[22] 王苏民,窦鸿身. 中国湖泊志. 北京:科学出版社,1998:537.

[23] 彭志忠. X 射线分析简明教程. 北京: 地质出版社,1982.

[24] Grim RE, Johns WD. Clay mineral investigation of sediments in the northern Gulf of Mexico. Clays and Clay Minerals, 1954, $2: 81-103$.

[25] Biscaye PE. Mineralogy and sedimentation of recent deep-sea clay in the Atlantic Ocean and adjacent seas and oceans. Bulletin of the Geological Society of America, 1965, 76(7) : 803-831.

[26] 孙千里,肖举乐. 岱海沉积物粒度特征及其古环境意义. 海洋地质与第四纪地质,2001,21(1):93-95.

[27] 张瑞瑾. 河流泥沙动力学. 北京: 中国水利水电出版社,1998.

[28] 汪海斌,陈发虎. 黄土高原西部地区黄土粒度的环境指示意义. 中国沙漠,2002,22(1):21-26.

[29] 雷祥义, 岳乐平. 陕西关中晚更新世黄土一古土壤序列特征及其记录的古环境变迁. 地质论评, 1997, 43 (5): $555-560$.

[30］张瑞虎, 刘 蹈, 黎 兵. 不同前处理方法和测量时间对泥炭中无机矿物颗粒粒度的影响. 沉积学报,2011,29(2): 374-380.

[31] 雷国良, 张虎才, 张文翔等. Mastersize 2000 型激光粒度仪分析数据可靠性检验及意义一一以洛川剖面 S4 层古土 壤为例. 沉积学报,2006,24(4):531-539.

[32] 尹秋珍, 郭正堂. 中国南方的网纹红土与东亚季风的异常强盛期. 科学通报,2006,51(2):186-193. 